\title{
Plasma Density Features Associated With Strong Convection in the Winter High-Latitude F Region
}

\author{
J. J. SOJKA, W. J. RAITT, AND R. W. SCHUNK \\ Center for Atmospheric and Space Sciences, Utah State University, Logan, Utah 84322
}

\begin{abstract}
We combined a simple plasma convection model with an ionospheric-atmospheric composition model in order to study the plasma density features associated with strong convection in the winter high-latitude $F$ region. Our numerical study produced time-dependent, three-dimensional, ion density distributions for the ions $\mathrm{NO}^{+}, \mathrm{O}_{2}{ }^{+}, \mathrm{N}_{2}{ }^{+}, \mathrm{O}^{+}, \mathrm{N}^{+}$, and $\mathrm{He}^{+}$. We covered the highlatitude ionosphere above $42^{\circ} \mathrm{N}$ magnetic latitude and at altitudes between 160 and $800 \mathrm{~km}$ for a time period of one complete day. From our study, we found the following: (1) For strong convection, the electron density exhibits a significant variation with altitude, latitude, longitude, and universal time. A similar result was obtained in our previous study dealing with a weak convection model. (2) For strong convection, ionospheric features, such as the main trough, the aurorally produced ionization peaks, the polar hole, and the tongue of ionization, are evident but they are modified in comparison with those found for slow convection. (3) For strong convection, the tongue of ionization is much more pronounced than for weak convection. (4) The polar hole that is associated with quiet geomagnetic activity conditions does not form when the plasma convection is strong. (5) For strong convection, a new polar hole appears in the polar cap at certain universal times. This new polar hole is associated with large downward, electrodynamic plasma drifts. (6) For strong convection, the main or mid-latitude electron density trough is not as deep as that found for a weak convection model. However, it is still strongly UT dependent. (7) The ionospheric parameters $N_{m} F_{2}, h_{m} F_{2}$, and the topside plasma density scale height exhibit an appreciable variation over the polar region at a given UT.
\end{abstract}

\section{INTRODUCTION}

Recently, we combined a plasma convection model and an ionospheric-atmospheric composition model in order to study the dynamics of the high-latitude $F$ region. The plasma convection model, which is based on the work of Volland [1978], includes the offset between the geographic and geomagnetic poles, the tendency of plasma to corotate about the geographic pole, and a dawn/dusk magnetospheric electric field mapped to a circular region in the ionosphere about a center offset by $5^{\circ}$ in the antisunward direction from the magnetic pole [Meng et al., 1977]. Equatorward of the circle the potential diminishes radially and varies inversely as the fourth power of sine magnetic co-latitude. The ionosphericatmospheric composition model takes account of solar EUV radiation, energetic particle precipitation, diffusion, thermospheric winds, electrodynamic drifts, energydependent chemical reactions, and magnetic storm induced neutral composition changes [cf. Schunk and Raitt, 1980]. For boundary conditions on the ion densities, we equated local production and loss rates at the lower boundary $(140 \mathrm{~km})$ and we assumed no flux of ions across our upper boundary $(800 \mathrm{~km})$. Earlier studies by Raitt et al. [1975] have indicated that a polar wind outflow has a negligible effect on the $\mathrm{O}^{+}$density profile at altitudes below $800 \mathrm{~km}$. The complete details concerning the combined plasma convection and ionosphericatmospheric models are given by Sojka et al. [1981a] and are not repeated here.

Our high-latitude $F$ region model produces timedependent, three-dimensional ion density distributions

Copyright $\odot 1981$ by the American Geophysical Union. for the ions $\mathrm{NO}^{+}, \mathrm{O}_{2}^{+}, \mathrm{N}_{2}^{+}, \mathrm{O}^{+}, \mathrm{N}^{+}$, and $\mathrm{He}^{+}$. Typically, we cover the high-latitude ionosphere above $42^{\circ} \mathrm{N}$ magnetic latitude and at altitudes between about 160 and $800 \mathrm{~km}$ for one complete day. In an initial application of the model, we studied the high-latitude winter $F$ region at solar minimum for low magnetic activity [Sojka et al., $1981 a, b$ ]. The main result we obtained was that highlatitude ionospheric features, such as the 'main trough,' the 'ionization hole,' the 'tongue of ionization,' the 'aurorally produced ionization peaks,' and the 'universal time effects,' are a natural consequence of the competition between the various chemical and transport processes known to be operating in the high-latitude ionosphere. We also obtained good agreement, both qualitatively and quantitatively, between our theoretical predictions and Atmosphere Explorer measurements of the variations of the ion composition with latitude and local time.

In this study, we modeled the high-latitude winter $F$ region for conditions corresponding to strong plasma convection. In addition, we adopted an asymmetric electric field pattern with a small convection cell in the dawnside polar region. This plasma convection pattern is in contrast to that adopted in our initial study, which corresponded to slow plasma convection with a symmetric electric field pattern. In section 2 , we describe our $F$ region model as it pertains to this study. In section 3 , we trace the history of a field tube of plasma as it traverses a typical convection trajectory. Section 4 is devoted to a discussion of the variation of the electron density with altitude, latitude, longitude, and universal time. In section 5 , we present the variation of some important ionospheric parameters, such as $N_{m} F_{2}, h_{m} F_{2}$, the $F$ region column content, and the topside plasma density scale height, while in section 6 we summarize our results. 


\section{F REGION MODEL}

Our model consists of two main components, a plasma convection model and an ionospheric-atmospheric composition model [Sojka et al., 1981a]. The model produces time-dependent, three-dimensional ion density distributions for the ions $\mathrm{NO}^{+}, \mathrm{O}_{2}{ }^{+}, \mathrm{N}_{2}{ }^{+}, \mathrm{O}^{+}, \mathrm{N}^{+}$, and $\mathrm{He}^{+}$. We cover the ionosphere above $42^{\circ} \mathrm{N}$ magnetic latitude and at altitudes between about 160 and $800 \mathrm{~km}$ for one complete day. A number of parameters are required as inputs to our model; the neutral composition, thermospheric wind, neutral temperature, electron temperature, EUV solar radiation spectrum, production due to auroral particle precipitation and resonantly scattered solar radiation, and a magnetospheric electric field. These parameters are used in the calculation of plasma convection, plasma diffusion, and photochemical processes, which in turn yield the ion density distributions. The model can describe different combinations of season, solar cycle, and geomagnetic activity levels. For this study we selected winter, solar maximum, and active magnetospheric conditions. As in our earlier model for quiet magnetospheric conditions, we maintained a constant cross-tail potential for the duration of our calculations (1.4 days).

The magnetospheric electric field is mapped into the ionosphere about the geomagnetic pole, which is offset from the northern geographic pole by $11.5^{\circ}$. In the polar cap region, defined by a circle of radius $18.5^{\circ}$ centered on a point $7.5^{\circ}$ antisunward of the geomagnetic pole, the electric field equipotentials are aligned sunward. Equatorward of the polar cap, the electric field diminishes and varies inversely as the fourth power of sine co-latitude in the magnetic frame. To simulate active magnetospheric conditions, a total cross-tail magnetospheric potential of $90 \mathrm{kV}$ was selected. In addition, an enhanced dusk cell pattern was adopted. Such an asymmetry in the convection electric field is commonly found in satellite electric field observations [Heppner, 1977] and in incoherent backscatter radar observations (J. Foster, private communications, 1980).

The adopted magnetospheric electric field needs to be combined with the tendency of the ionosphere to corotate in order to calculate the resultant plasma velocities. As described previously by Sojka et al. [1979a,b], when viewed in the magnetic quasi-inertial frame the convection velocities are UT independent, whereas in the geographic frame they are strongly UT dependent; a direct consequence of the offset and motion of the magnetic pole about the geographic pole. In Figure 1 we present 10 representative plasma drift trajectories in the magnetic quasi-inertial frame for our adopted asymmetric magnetospheric electric field pattern. Magnetic local time is shown as tick marks at hourly intervals on a circle representing $40^{\circ}$ magnetic latitude. Magnetic latitude increases linearly to the magnetic pole, shown by the vertical scale. The circulation times are tabulated for these 10 trajectories in the panel beneath the polar diagram. Field tubes of plasma following trajectories 1 and 2 appear to corotate; however, only trajectory 1 corresponds to corotation. Field tubes following trajectory 2 take 1.35 days to complete a full circulation owing to speed variations along its path. Field tubes of plasma following trajectories 3 and 4 execute motions which

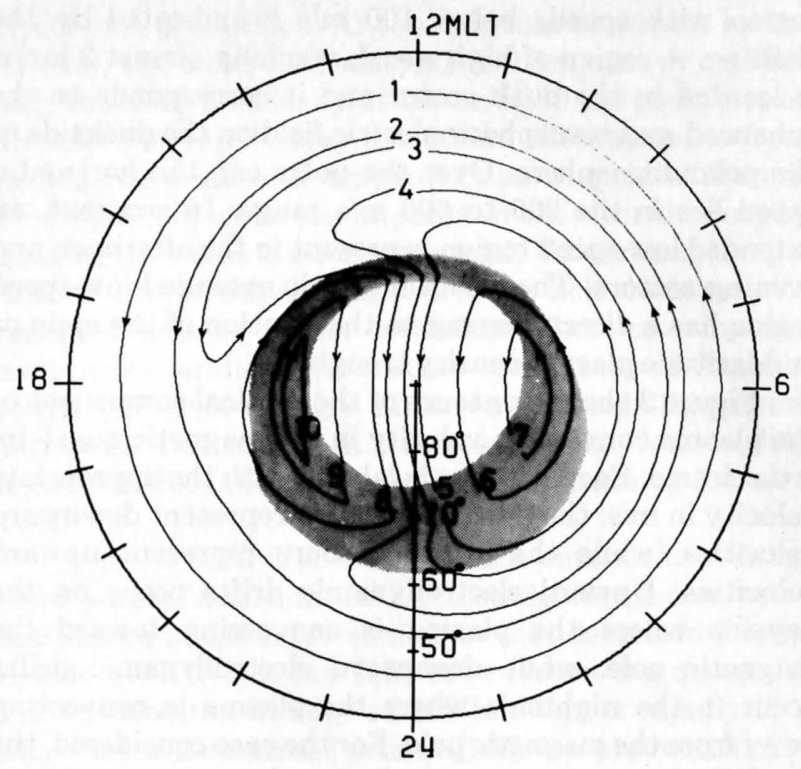

\begin{tabular}{|c|c|c|c|c|c|c|c|c|c|c|}
\hline LABEL & 1 & 2 & 3 & 4 & 5 & 6 & 7 & 8 & 9 & 10 \\
\hline $\begin{array}{c}\text { PEFIIOU } \\
\text { (OAYI }\end{array}$ & 1.06 & 1.35 & 1.32 & 0.79 & 0.58 & 0.25 & 0.15 & 0.27 & 0.11 & 0.06 \\
\hline
\end{tabular}

Fig. 1. Plasma drift trajectories in the magnetic quasi-inertial frame for a cross-tail magnetospheric electric potential of $90 \mathrm{kV}$. The shaded region corresponds to an auroral oval for $K_{p}=5$. Magnetic local time (MLT) is indicated by tick marks at hourly intervals and magnetic latitude is indicated on the vertical scale. The trajectories have been numbered in order to indicate circulation times, which are tabulated in the lower part of the figure.

result in a reversal of corotation in the afternoon sector. Field tubes following trajectories 5, 6, and 7 form a dawn cell rotating in a corotational sense, while field tubes following trajectories 8,9 , and 10 rotate in a counter corotation sense. In the centers of these two cells the plasma circulates extremely rapidly; 0.15 days for trajectory 7 and 0.06 days for trajectory 10 . An evening sector stagnation region is present in an extended region from 1800 to 2200 MLT and from $58^{\circ}$ to $62^{\circ}$ latitude. In our calculations, 44 trajectories were used to cover the ionosphere poleward of $42^{\circ}$ magnetic latitude.

Figure 1 also illustrates our adopted auroral oval, which corresponds to the $K_{p}=5$ auroral oval described by Comfort [1972]. When field tubes of plasma enter this region they are subjected to an ion production source resulting from energetic electron precipitation. The ion production rate profile that we adopted was taken from Knudsen et al. [1977] and corresponds to moderately active auroral electron precipitation. We did not include an ionization source due to low energy particle precipitation owing to the lack of information on precipitation fluxes over the high-latitude region. However, in our previous study [Sojka et al., 1981a], we investigated the extent to which such an ionization source could affect our results.

Since we have selected a large cross-tail magnetospheric potential of $90 \mathrm{kV}$, large plasma convection velocities can be expected in certain regions of the high-latitude ionosphere. This is shown in Figure 2, where contours of the horizontal plasma convection speed are plotted in the magnetic quasi-inertial frame. Each contour is labeled with its appropriate speed in $\mathrm{m} / \mathrm{s}$; the 
region with speeds below $100 \mathrm{~m} / \mathrm{s}$ is indicated by the shading. A region of high speed, reaching almost $2 \mathrm{~km} / \mathrm{s}$, is located in the dusk sector and it corresponds to the enhanced magnetospheric electric field on the duskside of the polar ionosphere. Over the polar cap the horizontal speed lies in the 200 to $600 \mathrm{~m} / \mathrm{s}$ range. In contrast, an extended low-speed region is present in the afternoon and evening sectors. The location of this extended low-speed region has a direct bearing on the location of the main or mid-latitude plasma density trough.

Figure 3 shows contours of the vertical component of the plasma convection velocity in the magnetic quasi- inertial frame. Each contour is labeled with the appropriate velocity in $\mathrm{m} / \mathrm{s}$; the dashed contours represent downward velocities, while the solid contours represent upward velocities. Upward electrodynamic drifts occur on the dayside where the plasma is convecting toward the magnetic pole, while downward electrodynamic drifts occur in the nightside where the plasma is convecting away from the magnetic pole. For the case considered, the vertical plasma drift ranges from $+50 \mathrm{~m} / \mathrm{s}$ to $-70 \mathrm{~m} / \mathrm{s}$. Vertical drifts near the extremes of this range have a pronounced effect on both the $F$ region peak electron density, $N_{m} F_{2}$, and the altitude of the peak, $h_{m} F_{2}$ [cf. Schunk et al., 1976].

In addition to the vertical motion associated with the $\mathbf{E} \times \mathbf{B}$ plasma drift, a thermospheric wind also induces a vertical plasma drift [cf. Rishbeth and Garriot, 1969; Watkins and Richards, 1979]. The importance of this additional vertical drift to the ion composition and to the maintenance of the nighttime high-latitude ionosphere was discussed by Sojka et al. [1981b]. In that study we adopted a modified form of the thermospheric wind pattern given by Murphy et al. [1976]. The Murphy et al. [1976] neutral wind is meridional everywhere, flowing towards the pole in the sunlit hemisphere and away from the pole in the dark hemisphere. The meridional flow reaches a maximum equatorward speed of $200 \mathrm{~m} / \mathrm{s}$ at 0100 local time (LT) and a maximum poleward speed of 60 $\mathrm{m} / \mathrm{s}$ at about $1300 \mathrm{LT}$. Near dawn and dusk the wind speed is extremely small. The small modification that we made to the Murphy et al. [1976] wind model was that we set the low-speed poleward wind to zero. This simplifica-

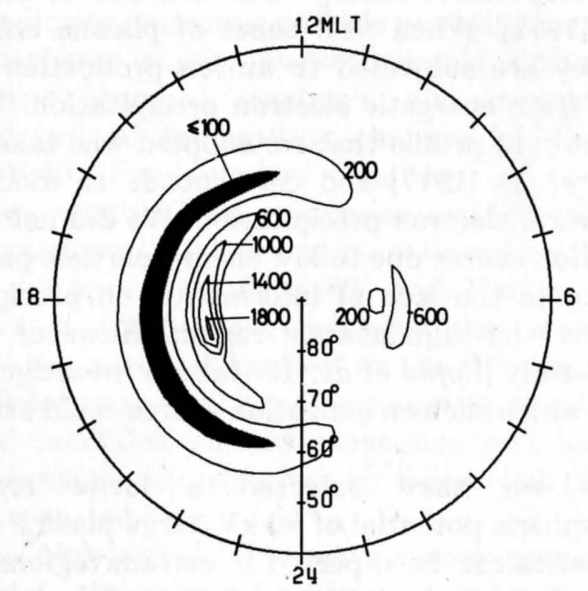

Fig. 2. Contours of horizontal plasma drift speeds in the magnetic quasi-inertial frame. The contours are labeled in units of $\mathrm{m} \mathrm{s}^{-1}$ and the shaded region corresponds to speeds below 100 $\mathrm{ms}^{-1}$.

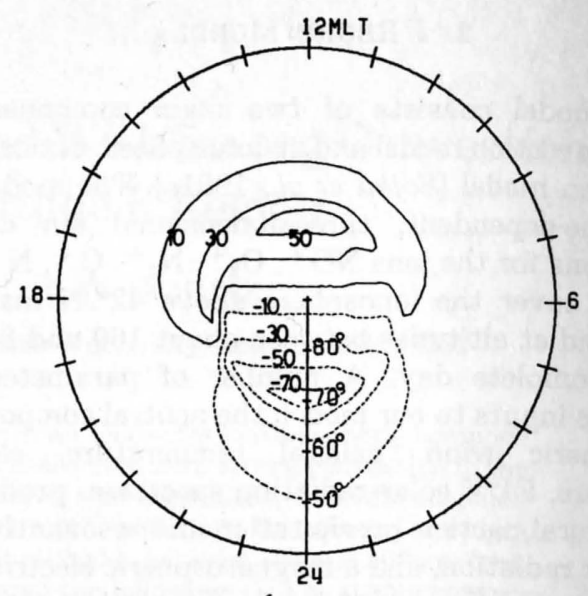

Fig 3. Contours of the vertical component of the electrodynamic plasma drift velocity displayed in the magnetic quasi-inertial frame. The solid contours correspond to upward drift and the dashed contours correspond to downward drift. The contours are labeled in units of $\mathrm{m} \mathrm{s}^{-1}$.

tion was motivated by the fact that thermospheric heating in the auroral oval acts to reduce or reverse this poleward wind. However, the equatorward wind on the nightside is enhanced by auroral oval heating, and therefore this equatorward wind must be included in order to obtain reliable predictions of ionospheric behavior on the nightside.

Figure 4 shows contours of the wind induced upward ionization drift in the magnetic quasi-inertial frame. The contours, which are labeled in $\mathrm{m} / \mathrm{s}$, display symmetry about an axis that extends from 1300 to 0100 MLT. Also, the wind induced upward ionization drift increases continuously with decreasing latitude over our latitude range, owing to its dependence on the magnetic field dip angle. Since the thermospheric wind is meridional in the geographic frame, it is UT dependent in the quasi-inertial magnetic frame of Figure 4. However, the UT variation is relatively small because the induced upward drift depends not only on the thermospheric wind but also on the magnetic field dip angle, which is UT independent in the magnetic frame.

In our model, the wind induced upward ionization drift is combined with the vertical component of the electrodynamic drift. A comparison of Figures 3 and 4 indicates that the wind induced and electrodynamic drifts oppose each other, producing a net downward drift in the polar cap and a net upward drift at mid-latitudes.

\section{Plasima in a Magnetic Field Tube}

As a field tube of plasma traverses a given trajectory it is subjected to different chemical and transport processes at different times. Also, at a given time the plasma in the field tube can be influenced by different physical processes at different altitudes. Consequently, before we present the variations of the electron density with altitude, latitude, longitude, and universal time, it is instructive to trace the temporal history of the plasma in a single field tube for one complete traversal of a trajectory.

Figure 5 shows the temporal history of a field tube of plasma following trajectory 3 in Figure 1 . The location of the field tube in the magnetic quasi-inertial frame is 


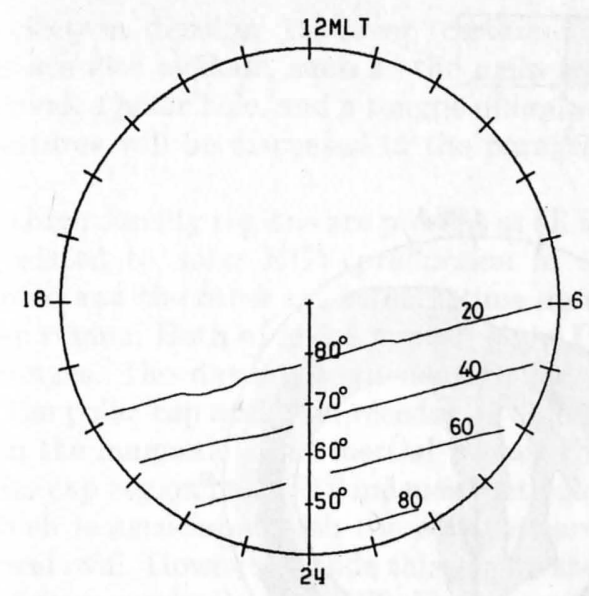

Fig. 4. Contours of the vertical component of the plasma drift velocity induced by the thermospheric wind displayed in the magnetic quasi-inertial frame. The contours correspond to upward drift and are labeled in units of $\mathrm{m} \mathrm{s}^{-1}$.

shown in the third panel of Figure 5. Magnetic local time is plotted as the solid line (left axis), while magnetic latitude is given by the dashed line (right axis). The four lower panels show the electron density versus UT at 160 , 260,360 , and $600 \mathrm{~km}$. On each of the four electron density curves, symbols have been plotted to denote every tenth density value. A study of the four panels indicates that the temporal history of the plasma in the flux tube is different at different altitudes, and at each altitude the plasma density exhibits a significant variation during the 32 hours that it takes the flux tube to complete a full cycle of trajectory 3 . These density variations are related to changes in the photochemical and transport processes that occur as the field tube of plasma moves along its trajectory. The changes related to transport are shown in the upper panel of Figure 5, where the field tube velocity in the quasi-inertial magnetic frame is plotted as a horizontal speed $(\mathrm{H}$, right axis) and a vertical velocity $(\mathrm{V}$, left axis), both in units of $\mathrm{m} / \mathrm{s}$. The changes relevant to photochemical processes are displayed by the shading, which indicates the times the field tube of plasma is in the auroral oval, and by the variation of the cosine of the solar zenith angle given in the second panel of Figure 5. When the cosine of the solar zenith angle is greater than zero, the field tube is sunlit and photoproduction is important.

Trajectory 3 was started at noon near the terminator. Subsequently, the field tube moves in a corotating fashion from about 0400 to 1200 UT. In this corotating phase, the electron density at all altitudes decreases owing to the continually increasing solar zenith angle. At about 1300 UT the field tube of plasma reverses direction and moves back toward noon. During this sunward motion, the electron density increases at all altitudes, but at $160 \mathrm{~km}$ the increase does not occur until 1500 UT. As the field tube moves back toward noon, its speed increases in the vicinity of the enhanced dusk convection cell. The upward plasma drift also increases, reaching 50 $\mathrm{m} / \mathrm{s}$. The entry of the field tube into the dayside auroral oval is accompanied by a rapid increase in the electron density at $160 \mathrm{~km}$, owing to auroral ion production. The electron density also increases at higher altitudes, but the increase is slower due to the dominance of transport effects over production. As the field tube of plasma leaves the oval, the electron density at $160 \mathrm{~km}$ decays rapidly, while the electron density at 260 and $360 \mathrm{~km}$ continues to increase. The rapid decay at $160 \mathrm{~km}$ simply results from the absence of auroral ion production coupled with ionic recombination, while the increase in the electron density at higher altitudes is a consequence of the upward component of the electrodynamic drift, which continues to transport ions to higher altitudes. Upon crossing the dawn-dusk meridian, the vertical component of the electrodynamic drift changes direction, driving the plasma downward. This results in a decrease in the electron density at 260,360 , and $600 \mathrm{~km}$ and a corresponding increase at $160 \mathrm{~km}$.

At about 2000 UT, the field tube enters the evening sector auroral oval and the electron density increases at all altitudes in a manner similar to that described for the dayside oval. When the field tube leaves the nocturnal oval, the electron density at $160 \mathrm{~km}$ decays rapidly to a value that is determined primarily by ion production due to resonantly scattered solar radiation. In contrast, at higher altitudes, where transport is important, the electron density is maintained for many hours by the wind induced upward ionization drift. Between about 3000 and 3400 UT the upward drift decreases, but ion production due to solar EUV radiation increases. This competition,
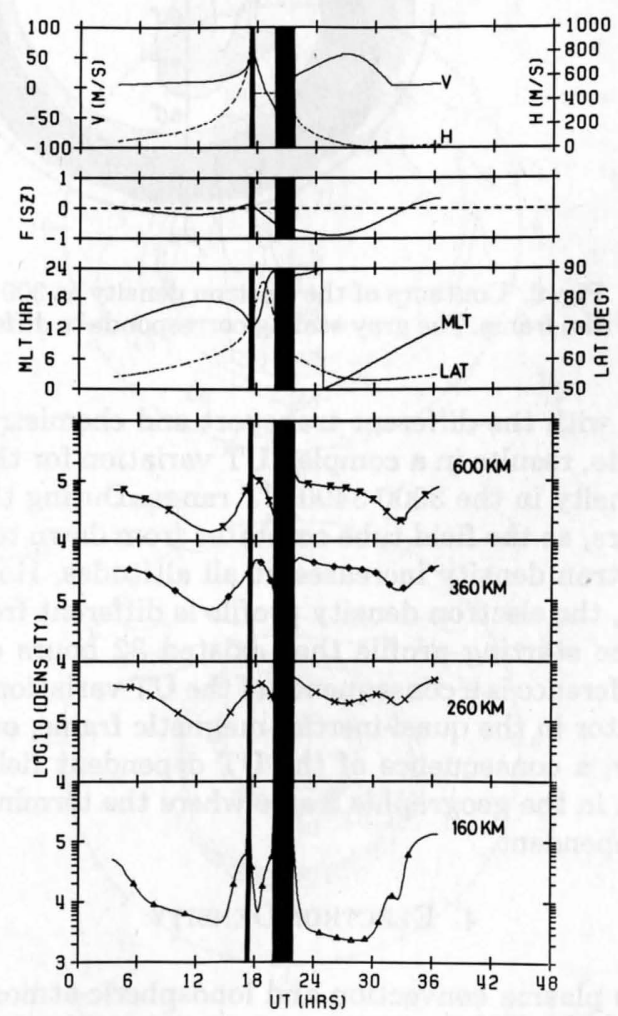

Fig. 5. The variation of electron density in a field tube of plasma convecting along trajectory 3 in Figure 1. In the lower four panels the density $\left(\mathrm{cm}^{-3}\right)$ at selected altitudes has been plotted against universal time. The top panel shows the variation of the vertical ( $V$, left scale) and horizontal ( $H$, right scale) plasma drift speeds. The second panel shows the variation of the cosine of the solar zenith angle. The third panel shows the variation of the magnetic local time (MLT, left scale) and magnetic latitude (LAT, right scale). The shaded regions indicate the times spent in the auroral oval by the plasma following trajectory 3 . 

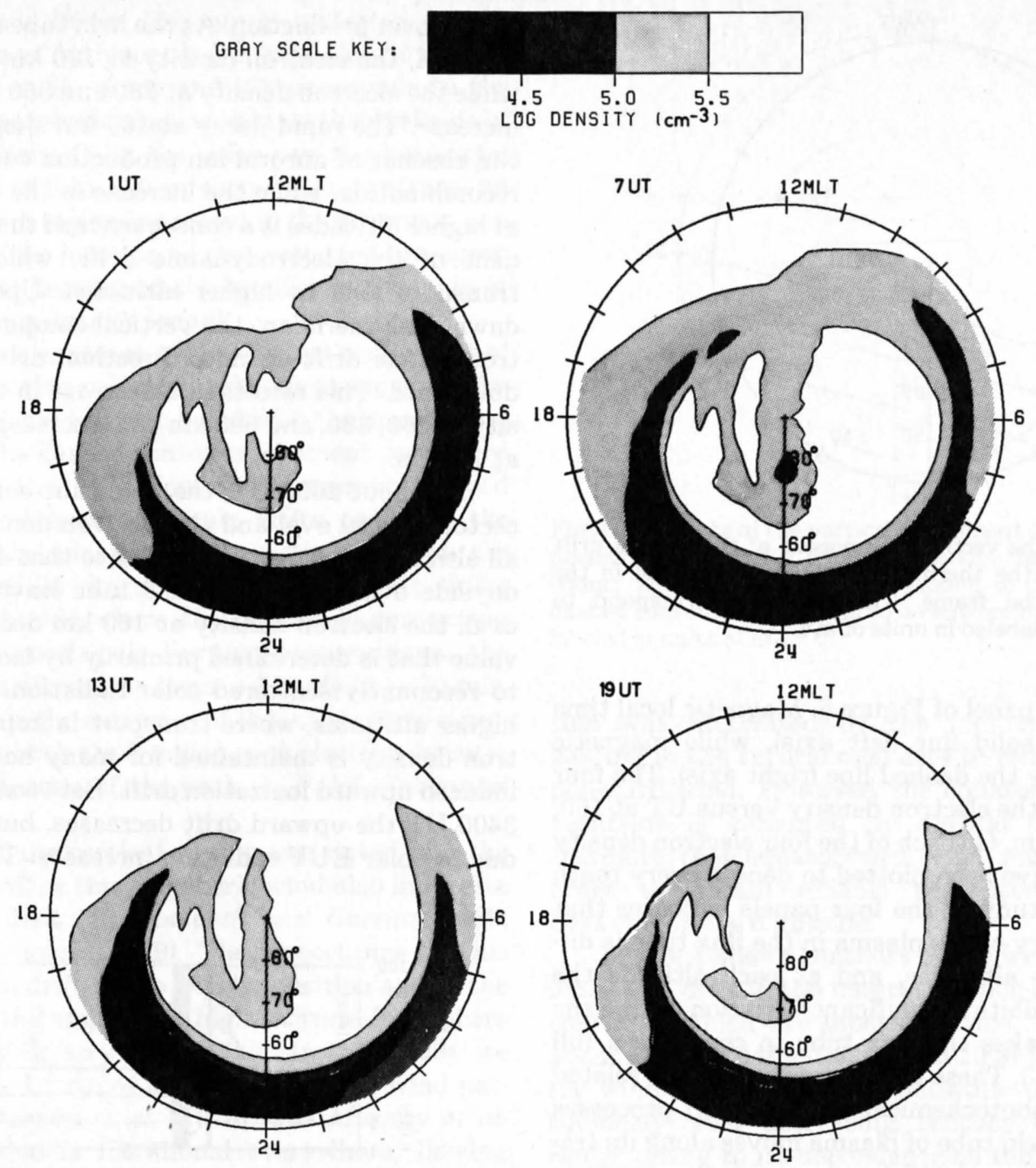

Fig. 6. Contours of the electron density at $300 \mathrm{~km}$ for four universal times displayed in the magnetic quasi- inertial frame. The gray scaling corresponds to different density levels, as indicated in the key.

coupled with the different transport and chemistry time constants, results in a complex UT variation for the electron density in the 3000-3400 UT range. During the last few hours, as the field tube corotates from dawn to noon, the electron density increases at all altitudes. However, at noon, the electron density profile is different from the noontime starting profile that existed 32 hours earlier. This difference is a consequence of the UT variation of the terminator in the quasi-inertial magnetic frame, or alternatively, a consequence of the UT dependent field tube location in the geographic frame where the terminator is UT independent.

\section{Electron DENSITY}

The plasma convection and ionospheric-atmospheric composition models were combined to generate altitude profiles of the various ion species over the area poleward of $42^{\circ} \mathrm{N}$ magnetic latitude, covering all local times for a 24-hour universal time period. In order to obtain adequate coverage in three-dimensional space and in universal time, the region of computation was divided up as follows. The altitude profiles consisted of ion densities computed every $4 \mathrm{~km}$ from 160 to $800 \mathrm{~km}$. A total of 44 drift trajectories was chosen to give adequate spatial coverage in the high-latitude region, and each trajectory was traversed 12 times, corresponding to 12 different UT starting times that were separated by 2 hours. Ion density profiles were stored at variable distances around the trajectory such that a typical trajectory at one UT had between 40 and 200 altitude profiles per ion species. The net result of the computations was a data base containing over 20,000 altitude profiles for each of the six ion species considered. The altitude profiles were distributed in magnetic latitude, MLT, and universal time. For convenience of presentation, these three parameters were divided into bins; the bin sizes were $3^{0}$ for magnetic latitude, 1 hour for MLT, and 2 hours for UT. Additional computational details are given by Sojka et al. [1981a].

Figure 6 shows contours of the electron density at $300 \mathrm{~km}$ for four universal times. The four UT's selected correspond to the geographic pole being near the dawn (0100 UT), noon (0700 UT), dusk (1300 UT), and midnight (1900 UT) magnetic meridians. In Figure 6, each plot is a polar diagram in MLT and magnetic latitude. The electron density variation is presented as gray scaled contour intervals, with the darkest shades corresponding to the lowest densities. Over the polar region, the electron density varies from about $10^{4}$ to $10^{6} \mathrm{~cm}^{-3}$. A particularly evident feature seen in Figure 6 is the marked UT variation 
of the electron density. However, certain ionospheric features are also evident, such as the main trough, the auroral oval, a polar hole, and a tongue of ionization, and these features will be discussed in the paragraphs that follow.

Two high density regions are present at all four UT's, one is related to solar EUV production in the sunlit hemisphere and the other is the nighttime auroral ovalpolar cap region. Both of these regions show UT dependent changes. The dayside high density region moves toward the polar cap and then recedes as the terminator moves in the magnetic quasi-inertial frame. The auroral oval-polar cap region has a UT independent equatorward edge which is associated with the equatorward edge of the auroral oval. However, inside this region the electron density varies markedly with UT. For example, at midnight and $80^{\circ}$ latitude, the electron density varies by more than an order of magnitude.

The two high density regions discussed above are, at times $(0100,1300$, and 1900 UT), linked by a tongue of high density in the dawn-noon sector. This tongue of ionization results from the poleward convection of dayside, high density field tubes (see Figure 1). The preference for a pre-noon tongue over a post-noon tongue is a consequence of the higher convection speeds in this sector (see Figure 2). With higher convection speeds the field tubes of plasma can convect further during a typical 'decay' time. For weak convection [Sojka et al., 1981a], on the other hand, field tubes of plasma travel a much shorter distance during this decay time, resulting in a much less pronounced tongue of ionization.

An ionospheric feature that is commonly associated with the winter polar ionosphere is the so-called 'polar hole' [Brinton et al., 1978]. This is a region where the plasma density at $300 \mathrm{~km}$ falls below $10^{3} \mathrm{~cm}^{-3}$ during quiet geomagnetic activity conditions. This polar hole region is located in the nightside polar cap adjacent to the poleward edge of the auroral oval in the midnight-dawn MLT sector. In a recent model study [Sojka et al., 1981a], we have shown that the Brinton et al. polar hole results from slow antisunward convection across the dark polar cap in combination with ordinary ionic recombination. In that model study, the plasma at $300 \mathrm{~km}$ had sufficient time to decay to a value as low as $5 \times 10^{2} \mathrm{~cm}^{-3}$, which was the level maintained by resonantly scattered solar radiation.

It is evident from Figure 6 that the Brinton et al. polar hole does not form during more active geomagnetic conditions, owing to the much shorter transit times across the polar cap. The electron density in the location of the Brinton et al. polar hole remains above $3 \times 10^{4}$ $\mathrm{cm}^{-3}$ at all UT's. However, there is another region in the polar cap at 7 UT that contains relatively low electron densities. In contrast to the low geomagnetic activity polar hole, which is due to chemical loss processes acting over a long time period, the high geomagnetic activity polar hole shown in Figure 6 results from the large downward transport velocities that exist in this region (see Figures 3 and 4).

Another ionospheric feature that is known to occur in the winter polar ionosphere is the 'mid-latitude' or 'main' electron density trough. This trough is a region of low electron density situated just equatorward of the nocturnal auroral oval. It is generally apparent at all univer-
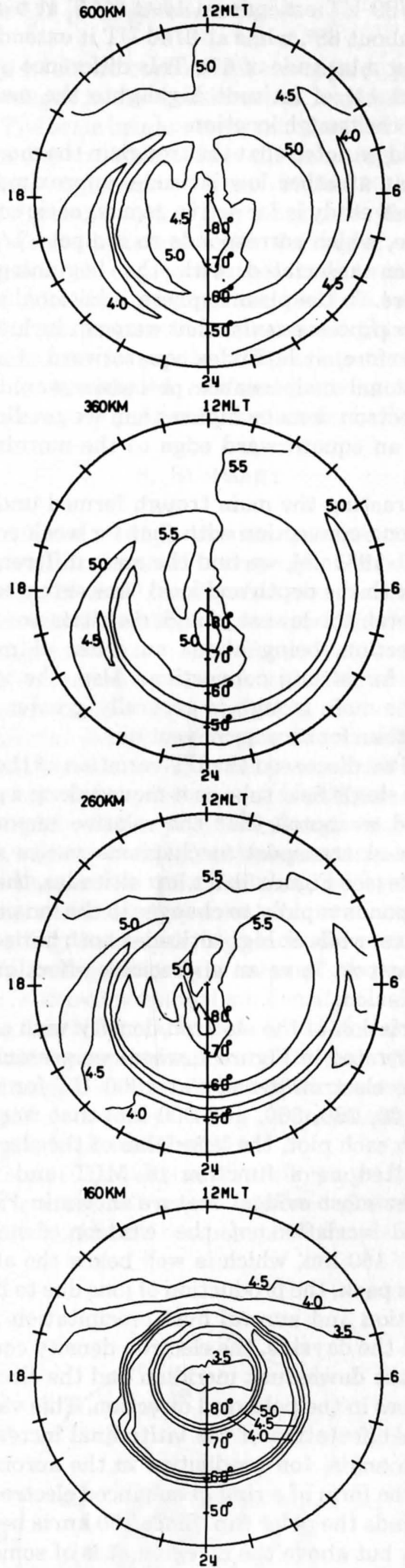

Fig. 7. Contours of the electron density at 1000 UT for four altitudes displayed in the magnetic quasi-inertial frame. The contours are labeled in units of $\log _{10} n_{e}\left(\mathrm{~cm}^{-3}\right)$.

sal times (see Figure 6), but its depth and local time extent are strongly UT dependent. At $0100 \mathrm{UT}$, it is deepest in the morning sector; at $1300 \mathrm{UT}$, it is deeper in the evening sector than in the morning sector; while in the middle of this UT range the trough tends to be more symmetrical about midnight. The trough in the afternoon 
sector at 0700 UT extends to $1500 \mathrm{MLT}$ at a magnetic latitude of about $68^{\circ}$, while at $0100 \mathrm{UT}$ it extends to only $1900 \mathrm{MLT}$ at a latitude of $57^{\circ}$. This difference of 4 hours of MLT and $11^{\circ}$ of latitude highlights the marked UT variation of the trough location.

It should be noted that the trough in the morning sector occurs at a rather low latitude, approximately $50^{\circ}$. Although this study is for active geomagnetic conditions, this latitude, which corresponds to a dipole ' $L$ ' value of 2.4 , is often associated with the beginning of the plasmasphere. In the plasmasphere, additional nocturnal maintenance processes exist that were not included in our model. Therefore, at latitudes equatorward of about $50^{\circ}$ these additional maintenance processes would tend to keep the electron density higher than we predict, acting to produce an equatorward edge to the morning sector trough.

In contrasting the main trough formed under conditions of strong convection with that for weak convection [Sojka et al., 1981a,b], we find the main differences to be connected with the depth and local time extent at a given UT. In general, the lowest trough densities are found for weak convection, being about an order of magnitude lower than for strong convection. Also, the local time extent of the main trough is generally greater for weak convection than for strong convection.

Earlier, we discussed the UT variation of the electron density in a single field tube as it moves along a given trajectory, and we noted that the relative importance of chemistry and transport mechanisms varies markedly with altitude (see Figure 5). At low altitudes, the electron density responds rapidly to changes in the ion production and loss rates, while at high altitudes both horizontal and vertical transport have an appreciable effect on the UT density variation.

The variation of the electron density with altitude is further illustrated in Figure 7, where we present contour plots of the electron density at 1000 UT for the same altitudes $(160,260,360$, and $600 \mathrm{~km})$ that were used in Figure 4. In each plot, the logarithm of the electron density is plotted as a function of MLT and magnetic latitude. The most evident feature shown in Figure 7 is the marked variation of the electron density with altitude. At $160 \mathrm{~km}$, which is well below the altitude of the $F$ region peak, the production of ions due to both solar EUV radiation and auroral oval precipitation is clearly evident. On the dayside, the electron density contours lie parallel to the dawn-dusk meridian and the electron density decreases in the poleward direction. This variation is simply a manifestation of the latitudinal increase in the solar zenith angle. Ion production in the auroral oval is evident in the form of a ring of enhanced electron density that surrounds the polar cap. Since $160 \mathrm{~km}$ is below the $F$ region peak but above the $E$ region, it is of some interest that the electron density is almost purely dependent on the ion chemistry and that electrodynamic transport is relatively unimportant.

At $260 \mathrm{~km}$, on the other hand, the effects of plasma transport are evident in a number of ways. First, the contour labeled 5.5 in the sunlit hemisphere, which corresponds to one of the 'solar zenith' contours at $160 \mathrm{~km}$, is now twisted such that it is parallel to the $0700-1900$ MLT meridian. This twisting results from the tendency of the ionosphere to corotate at these lower latitudes. Also, the high density region in the polar cap is significantly broader than it is at $160 \mathrm{~km}$, owing to plasma transport. This is particularly true in the morning sector where flux tubes leave the auroral oval and convect sunward on the equatorward edge, thus broadening the region of high density. High density plasma is also convected out of the auroral oval and into the dayside polar cap. This auroral plasma combines with the dayside plasma that has convected in an antisunward direction across the polar cap, thus forming a tongue of ionization.

At 360 and $600 \mathrm{~km}$, the progressive dominance of transport phenomena is evident. The dayside feature associated with the solar zenith angle has all but disappeared. Likewise, at both 360 and $600 \mathrm{~km}$, the shape of the enhanced density region associated with auroral oval production is significantly modified from that found at $160 \mathrm{~km}$. Also, at $360 \mathrm{~km}$ plasma transport has acted to produce a well-defined trough minimum between 1900-2000 MLT. The maintenance of the higher electron densities at later local times being due to the wind induced, upward ionization drift.

\section{REDUCED IONOSPHERIC PARAMETERS}

A number of ionospheric parameters have been frequently used to describe the $F$ region. In this section we will discuss four such parameters, namely $h_{m} F_{2}, N_{m} F_{2}$, the $F$ region column content, and the topside plasma scale height. In deriving these parameters, the model electron density was used rather than a particular ion density.

Figure 8 shows contours of the altitude of the $F$ region peak density in the magnetic quasi-inertial frame for 1000 UT. The contours are labeled in $\mathrm{km}$. Although only one UT is shown, the UT variation of $h_{m} F_{2}$ is small, being on the order of $10 \mathrm{~km}$ at a given location. In Figure 8 , the altitude of the peak density varies from 250 to 380 $\mathrm{km}$, with there being two distinct regions where $h_{m} F_{2}$ is large. One region, near the noon meridian at $80^{\circ}$, is associated with a large upward electrodynamic drift, while the other region, the nightside mid-latitude ionosphere, is associated with the upward plasma drift induced by the thermospheric wind. The minimum values

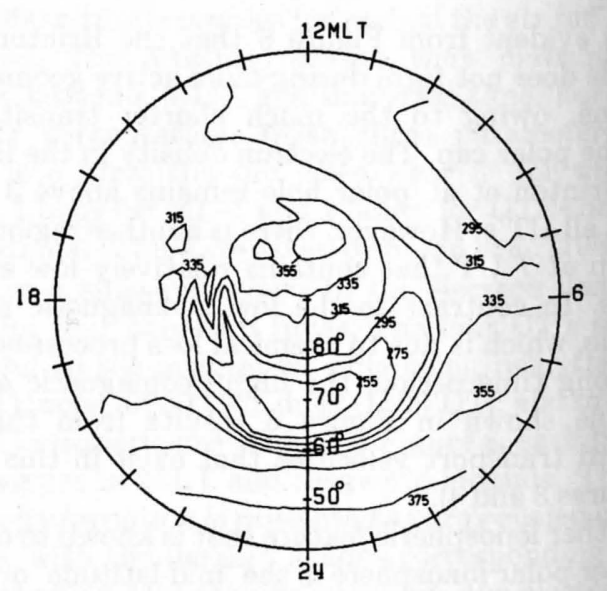

Fig. 8. Contours of $h_{m} F_{2}$ at 1000 UT displayed in the magnetic quasi-inertial frame. The contours are labeled in units of kilometers. 
PEAK DENSITIES

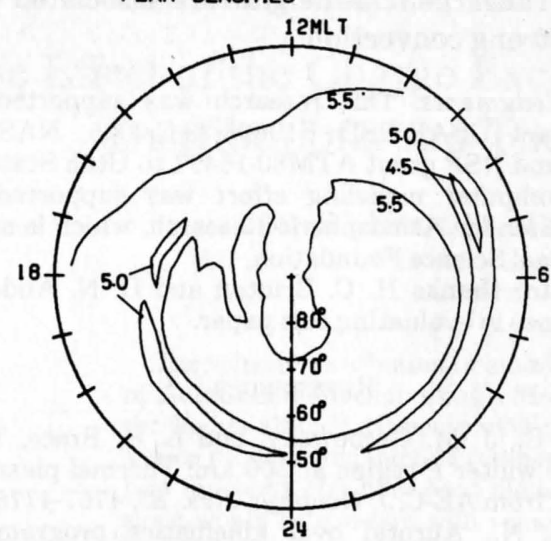

COUMN CONTENT

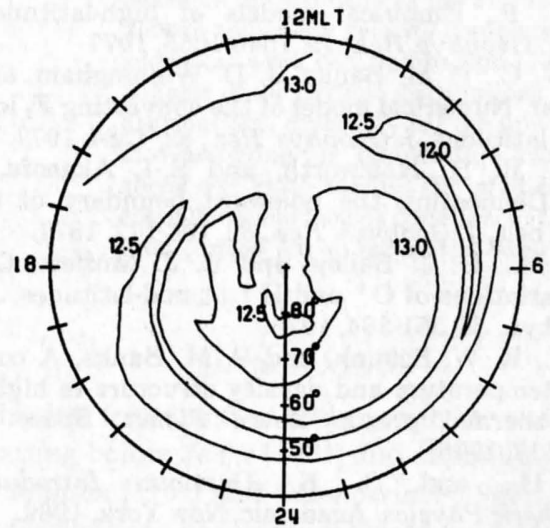

Fig. 9. Contours of $N_{m} F_{2}$ (top panel) and column content (bottom panel) at 1000 UT displayed in the magnetic quasi-inertial frame. The peak density contours are labeled in units of $\log _{10} n_{e}$ $\left(\mathrm{cm}^{-3}\right)$, while the contours of the column content are labeled in units of $\log _{10}\left(\mathrm{~cm}^{-2}\right)$.

of $h_{m} F_{2}$, on the other hand, are found near the midnight meridian at $\mathbf{7 0 ^ { \circ }}$. This location corresponds to the region where the maximum downward electrodynamic drifts occur. In this regard, it should be noted that the $h_{m} F_{2}$ contours display features that are similar to those found in the contours of the vertical velocities associated with the electrodynamic and wind induced drifts (see Figures 3 and 4). The close similarity between these sets of contours emphasizes the importance of vertical transport in determining the altitude of the $F$ region peak electron density. The lack of a significant UT variation in $h_{m} F_{2}$ is a further indication of this relationship, since in the magnetic quasi-inertial frame the vertical velocities are almost UT independent.

Figure 9 shows contours of the logarithm of $N_{m} F_{2}$ (upper panel) and the logarithm of the $F$ region column content (lower panel) at 1000 UT. The column content was computed as the density integral from 160 to $800 \mathrm{~km}$. The most evident feature to emerge from a comparison of the two panels is the similarity of the two contour patterns. Although both $N_{m} F_{2}$ and the column content exhibit a significant UT variation, this similarity is present at all universal times. The obvious inference from the similarity of $N_{m} F_{2}$ and the column content is that the bulk of the column content is contained in a region near the $F$ region peak. With regard to the variation of $N_{m} F_{2}$ with UT, it follows the general pattern of change shown in the four UT plots given in Figure 6 for an altitude of $300 \mathrm{~km}$.

Figure 10 shows contours of the topside plasma density scale height in the quasi-inertial magnetic frame for 1000 UT. The scale heights were calculated in the altitude range from 600 to $800 \mathrm{~km}$. Over the polar region the scale height varies significantly, from 190 to $330 \mathrm{~km}$. A comparison of Figure 10 with Figure 2 indicates that the large scale heights are associated with the region of high convection speeds. Strong convection leads to an enhanced ion-neutral frictional heating rate, which in turn leads to elevated ion temperatures and increased plasma density scale heights. As far as the UT variation of the scale height is concerned, the contour patterns obtained at other UT's are very similar to that shown in Figure 10.

\section{SUMMARY}

We combined a simple plasma convection model with an ionospheric-atmospheric composition model in order to study the plasma density features associated with strong convection in the winter high-latitude $F$ region. The details of our time-dependent ionosphericatmospheric composition model, including ion chemistry and transport equations, are given by Schunk and Raitt [1980], while the details concerning the combined plasma convection and ionospheric-atmospheric models are given by Sojka et al. [1981a]. In a model calculation a field tube of plasma is followed as it moves along a convection trajectory through a moving neutral atmosphere. Altitude profiles of the ion densities are obtained by solving the appropriate continuity, momentum, and energy equations including numerous high-latitude processes. The result of following many field tubes of plasma is a timedependent, three-dimensional ion density distribution for each of the ions $\mathrm{NO}^{+}, \mathrm{O}_{2}{ }^{+}, \mathrm{N}_{2}{ }^{+}, \mathrm{O}^{+}, \mathrm{N}^{+}$, and $\mathrm{He}^{+}$. The high-latitude ionosphere above $42^{\circ} \mathrm{N}$ magnetic latitude and at altitudes between 160 and $800 \mathrm{~km}$ was covered for one complete day by using a constant cross-tail magnetospheric potential of $90 \mathrm{kV}$.

From our study we found the following:

1. For a cross-tail magnetospheric electric potential of $90 \mathrm{kV}$, the horizontal component of the plasma convec-

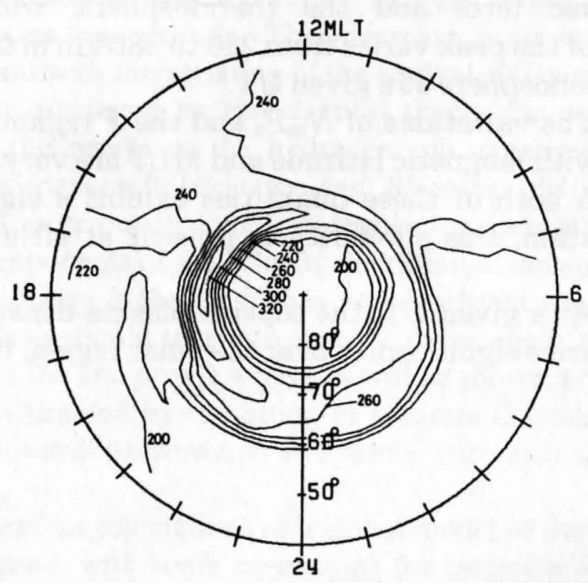

Fig. 10. Contours of the plasma density scale height at 1000 UT displayed in the magnetic quasi-inertial frame. The contours are labeled in units of kilometers. 
tion velocity in the ionosphere approaches $2 \mathrm{~km} / \mathrm{s}$ and the vertical component ranges from $+50 \mathrm{~m} / \mathrm{s}$ to $-70 \mathrm{~m} / \mathrm{s}$. Vertical drifts near the extremes of this range have a pronounced effect on both $N_{m} F_{2}$ and $h_{m} F_{2}$.

2. As a field tube of plasma traverses a given trajectory it is subjected to different chemical and transport processes at different times. Also, at a given time the plasma in the field tube can be influenced by different physical processes at different altitudes.

3. As was found in our previous study dealing with weak convection [Sojka et al., 1981a], for strong convection the electron density exhibits a significant variation with altitude, latitude, longitude, and universal time.

4. For strong convection, certain ionospheric features, such as the main trough, the aurorally produced ionization peaks, the polar hole, and the tongue of ionization, are evident but they are modified in comparison with those found for slow convection.

5. For strong convection, the tongue of ionization that extends across the polar cap is much more pronounced than for slow convection. This tongue of ionization is highly UT dependent.

6. The polar hole found by Brinton et al. [1978] during quiet geomagnetic activity conditions, which results from weak antisunward convection across the dark polar cap in combination with ordinary ionic recombination, does not form for the more active geomagnetic conditions considered in this study, owing to the much shorter transit times across the polar cap.

7. For strong convection, a new polar hole appears in the polar cap at certain universal times. This new polar hole is associated with large, downward, electrodynamic plasma drifts.

8. For strong convection, the main or mid-latitude electron density trough is generally not as deep as that found for quiet geomagnetic activity conditions. However, in both cases the depth and extent of the trough are strongly UT dependent. At certain UT's the trough is deeper in the morning sector than in the evening sector, while at other UT's the reverse is true. At still other times, the trough tends to be more symmetrical about midnight.

9. The altitude of the peak electron density exhibits a significant variation with magnetic latitude and MLT, owing to the vertical plasma drifts induced by the electrodynamic force and the thermospheric wind. The altitude of the peak varies from 250 to $380 \mathrm{~km}$ in the highlatitude ionosphere at a given UT.

10. The variations of $N_{m} F_{2}$ and the $F$ region column content with magnetic latitude and MLT are very similar. Although both of these quantities exhibit a significant UT variation, this similarity is present at all universal times.

11. At a given UT, the topside plasma density scale height varies significantly over the polar region, from 190 to $330 \mathrm{~km}$. The large scale heights are associated with the
regions of strong convection.

Acknowledgments. This research was supported by Air Force contract USAF/ESD F19628-79-C-0025, NASA grant NAGW-77, and NSF grant ATM80-15497 to Utah State University. The computer modeling effort was supported by the National Center for Atmospheric Research, which is sponsored
by the National Science Foundation.

The Editor thanks H. C. Brinton and D. N. Anderson for their assistance in evaluating this paper.

\section{REFERENCES}

Brinton, H. C., J. M. Grebowsky, and L. H. Brace, The highlatitude winter $F$ region at $300 \mathrm{~km}$ : Thermal plasma observations from AE-C, J. Geophys. Res., 83, 4767-4776, 1978.

Comfort, R. N., Auroral oval kinematics program, NASA Rep. CR-61373, 1972.

Heppner, J. P., Empirical models of high-latitude electric fields, J. Geophys. Res., 79, 1046-1055, 1977.

Knudsen, W. C., P. M. Banks, J. D. Winningham, and D. M. Klumpar, Numerical model of the convecting $F_{2}$ ionosphere at high latitudes, J. Geophys. Res., 82, 4784, 1977.

Meng, C.-I., R. H. Holzworth, and S.-I. Akasofu, Auroral circle-Delineating the poleward boundary of the quiet auroral belt, J. Geophys. Res., 82, 164-172, 1977.

Murphy, J. A., G. J. Bailey, and R. J. Moffett, Calculated daily variations of $\mathrm{O}^{+}$and $\mathrm{H}^{+}$at mid-latitudes, J. Atmos. Terr. Phys., 38, 351-364, 1976.

Raitt, W. J., R. W. Schunk, and P. M. Banks, A comparison of the temperature and density structure in high and low speed thermal proton flows, Planet. Space Sci., 23, 1103-1117, 1975.

Rishbeth, H., and O. K. Garriott, Introduction to Ionospheric Physics, Academic, New York, 1969.

Schunk, R. W., and W. J. Raitt, Atomic nitrogen and oxygen ions in the daytime high-latitude $F$ region, $J$. Geophys. Res., 85, 1255-1272, 1980.

Schunk, R. W., P. M. Banks, and W. J. Raitt, Effects of electric fields and other processes upon the nighttime high latitude F layer, J. Geophys. Res., 81, 3271-3282, 1976.

Sojka, J. J., W. J. Raitt, and R. W. Schunk, Effect of displaced geomagnetic and geographic poles on highlatitude plasma convection and ionospheric depletions, $J$. Geophys. Res., 84, 5943-5951, $1979 a$.

Sojka, J. J., W. J. Raitt, and R. W. Schunk, High latitude plasma convection: Predictions for Eiscat and Sondre Stromfjord, Geophys. Res. Lett., 6, 877-880, 1979 .

Sojka, J. J., W. J. Raitt, and R. W. Schunk, A theoretical study of the high-latitude winter $F$ region at solar minimum for low magnetic activity, J. Geophys. Res., 86, 609-621, $1981 a$.

Sojka, J. J., W. J. Raitt, and R. W. Schunk, Theoretical predictions for ion composition in the high-latitude winter $F$ region for solar minimum and low magnetic activity, $J$. Geophys. Res., 86, 2206-2216, $1981 b$.

Volland, H., A model of the magnetospheric electric convection field, J. Geophys. Res., 83, 2695-2699, 1978.

Watkins, B. J., and P. G. Richards, A theoretical investigation of the role of neutral winds and particle precipitation in the formation of the auroral $F$ region ionosphere, J. Atmos. Terr. Phys., 41, 179-187, 1979.

(Received February 17, 1981; revised April 15, 1981; accepted April 17, 1981.) 